\title{
Performance Analysis of a Decoding Algorithm for Algebraic Geometry Codes
}

Jensen, Helge Elbrønd; Nielsen, Rasmus Refslund; Høholdt, Tom

Published in:

Information Theory, 1998. Proceedings. 1998 IEEE International Symposium on

Link to article, DOI:

10.1109/ISIT.1998.708983

Publication date:

1998

Document Version

Publisher's PDF, also known as Version of record

Link back to DTU Orbit

Citation (APA):

Jensen, H. E., Nielsen, R. R., \& Høholdt, T. (1998). Performance Analysis of a Decoding Algorithm for Algebraic Geometry Codes. In Information Theory, 1998. Proceedings. 1998 IEEE International Symposium on IEEE. https://doi.org/10.1109/ISIT.1998.708983

\section{General rights}

Copyright and moral rights for the publications made accessible in the public portal are retained by the authors and/or other copyright owners and it is a condition of accessing publications that users recognise and abide by the legal requirements associated with these rights.

- Users may download and print one copy of any publication from the public portal for the purpose of private study or research.

- You may not further distribute the material or use it for any profit-making activity or commercial gain

- You may freely distribute the URL identifying the publication in the public portal 


\section{Performance Analysis of a Decoding Algorithm for Algebraic Geometry Codes}

\author{
H.Elbrond Jensen \\ Dept. of Mathematics \\ Technical University of Denmark \\ Bldg.303 \\ DK-2800 Lyngby Denmark
}

Email h.elbrond.jensen@mat.dtu.dk
R.Refslund Nielsen

Dept. of Mathematics

Technical University of Denmark Bldg. 303

DK-2800 Lyngby Denmark

Email stud-rrn@mat.dtu.dk

\author{
T.Hoeholdt \\ Dept.of Mathematics \\ Technical University of Denmark \\ Bldg.303 \\ DK-2800 Lyngby Denmark \\ Email tom@mat.dtu.dk
}

Abstract - We analyse the known decoding algorithms for algebraic geometry codes in the case where the number of errors is greater than or equal to $\left\lfloor\left(d_{F R}-1\right) / 2\right\rfloor+1$, where $d_{F R}$ is the Feng-Rao distance.

\section{INTRODUCTION}

The fast decoding algorithm for one-point algebraic geometry codes of Sakata, Elbrønd Jensen, and Høholdt [1] decodes any error pattern of weight up to $\left\lfloor\left(d_{F R}-1\right) / 2\right\rfloor$ where $d_{F R}$ is the so-called Feng-Rao distance of the code. In this paper we analyse the performance of the decoding algorithm, when the number of errors is greater than or equal to $\left\lfloor\left(d_{F R}-1\right) / 2\right\rfloor+1$.

\section{The CODEs AND THE DECODING Algorithm}

Let $P_{1}, P_{2}, \ldots, P_{n}, Q$ be $F_{q}$-rational points on a nonsingular absolutely irreducible curve $\chi$ of genus $g$ defined over $F_{q}$. We consider an algebraic geometry code $C_{m}$ of type $C_{L}(D, G)^{\perp}=$ $C_{\Omega}(D, G)$, where $D=P_{1}+P_{2}+\ldots+P_{n}$ and $G=m Q$.

If $f \in R$ and $\underline{y} \in F_{q}^{n}$ we define the syndrome $S_{\underline{y}}(f)$ to be

$$
S_{\underline{y}}(f)=\sum_{i=1}^{n} y_{i} f\left(P_{i}\right)
$$

so we have $\underline{y} \in C \Longleftrightarrow S_{\underline{y}}(f)=0$ for all $f$ such that $\rho(f) \leq m$. In the decoding situation we receive a vector $y$ which is the sum of a codeword $\underline{c}$ and an error vector $\underline{e}$. We have $S_{\underline{\underline{e}}}(f)=$ $S_{\underline{y}}(f)$ if $\rho(f) \leq m$, so the syndromes $S_{\underline{e}}(f)$ can be calculated directly from the received word if $\rho(f) \leq m$.

If $\tau$ is the Hamming weight of $\mathrm{e}$ then it is well known e.g. [1] or [2] that if one knows the syndromes $S_{\underline{e}}(f)$ where $\rho(f) \leq 2(\tau+2 g)-1$ then the error vector can be easily found. The objective of the decoder is therefore to determine the syndromes $S_{\underline{\underline{e}}}(f)$ where $m<\rho(f) \leq 2(\tau+2 g)-1$.

The decoding algorithm is a version of Sakata's generalization of the Berlekamp-Massey algorithm.

This algorithm indeed solves the decoding problem when $\tau \leq\left\lfloor\left(d_{F R}-1\right) / 2\right\rfloor$ (with $\tau$ being the number of errors). See [2] or [1].

\section{THE RESULTS}

Let $P_{1}, \ldots, P_{\tau}$ be the error points. We call these independent, if they give independent conditions on a function passing through these points, or equivalently that

$$
\mathrm{L}\left(\rho Q-\left(P_{1}+\cdots+P_{\tau}\right)\right)=0 \text { for } \rho \leq \rho_{\tau}
$$

Theorem 1 If $m \geq 4 g-2, \tau>\left\lfloor\left(d_{F R}-1\right) / 2\right\rfloor$, and the error points are independent then the algorithm fails.
The algorithm can fail by either giving no answer or a wrong answer, and indeed both cases can occur.

When $m<4 g-2$ the situation is different. We have developed a fairly simple procedure to determine the performance of the decoding algorithm in this case also. We mention that for the Hermitian curve over $F_{r^{2}}$ given by the equation

$$
x^{r+1}+y^{r}+y=0
$$

which has genus $g=\frac{r(r-1)}{2}$ and $r^{3} F_{r^{2}}$-rational points we can often do much better than predicted by the Feng-Rao bound.

If $r=4$ we can get a $(64,57,4)$-code over $F_{16}$, but two independent errors are always decoded correctly.

If $r=8$ we get a $(512,476,9)$-code over $F_{64}$, but here one can always decode 10 independent errors correctly. By similar considerations we can explain the results presented by $\mathrm{O}^{\prime}$ Sullivan in [3].

The error points can fail to be independent in different ways. If we look at the case where $\tau=\left\lfloor\left(d_{F R}-1\right) / 2\right\rfloor+1$ and

$$
\mathrm{L}\left(\rho Q-\left(P_{1}+\cdots+P_{\tau}\right)\right)=0 \text { for } \rho<\rho_{\tau}
$$

but $\mathrm{L}\left(\rho_{\tau} Q-\left(P_{1}+\cdots+P_{\tau}\right)\right) \neq 0$, we have the following two theorems:

Theorem 2 The function in $F_{M}$ with lowest poleorder $\rho$ at $Q$ is an element of $L\left(\rho Q-\left(P_{1}+\cdots+P_{T}\right)\right)$ for at least $(q-1)^{\tau-1}$ of the $(q-1)^{\tau}$ possible choices of the error values.

Theorem 3 The algorithm corrects $\tau=\left\lfloor\left(d_{F R}-1\right) / 2\right\rfloor+1$ dependent errors correctly in almost all cases.

The question whether a random selected set of points on a curve are independent or not seems difficult. We have some numerical evidence for conjecturing that (at least on a Hermitian curve) that the probability of getting independent points is $1-\frac{1}{q}$.

\section{REFERENCES}

[1] S. Sakata, H. Elbrønd Jensen and T. Høholdt: "Generalized Berlekamp-Massey Decoding of Algebraic-Geometric Codes up to half the Feng-Rao Bound"IEEE Trans. Inform. Theory, vol 41, no. 6, pp. 1762-1768, nov. 1995.

[2] M.E. O'Sullivan: "Decoding of Codes Defined by a single Point on a Curve" IEEE Trans. Inform. Theory, vol. 41, no. 6, pp. 1709-1719, nov. 1995.

[3] M.E. O'Sullivan: "Decoding Hermitian Codes beyond ( $d_{\min }-$ 1)/2" Proceedings of the 1997 IEEE International Symposium on Information Theory, June 20-July 4, 1997, Ulm, Germany. 